\title{
Metodología y ámbitos del Trabajo Social comunitario para impulsar cambios sociales sostenibles y autónomos en el complejo universo relacional en España en el siglo XXI
}

\section{Methodology and scope of community Social Work to promote sustainable social change in the complex autonomous relational universe in Spain in the XXI century}

\author{
Enrique Pastor Seller
}

\begin{abstract}
Resumen: La intervención comunitaria desde el Trabajo Social debe ofrecer respuestas a las necesidades, problemas y aspiraciones de manera integral con sus dificultades y oportunidades y, desde un enfoque estratégico y participativo, en concordancia con un marco teórico-conceptual y metodológico coherente. El artículo presenta el proceso metodológico vinculado con un trabajo social con comunidades, centrado en el modelo de desarrollo local estratégico, integral y sostenible, estructurado en torno a los procesos de caracterización y enlace con comunidad, análisis estratégico, formulación de intervención, implementación de propuesta de intervención y evaluación y/o reformulación del modelo de intervención. En cada una de los procesos se detalla metodología, objetivos, técnicas y competencias y habilidades profesionales requeridas. Para finalizar, se describen los ámbitos del trabajo social comunitario atendiendo a los sistemas de protección social, destacando objetivos, equipamientos y actuaciones a emprender en el trabajo social con comunidades en España.
\end{abstract}

Palabras clave: Trabajo social con comunidades. Cambio social. Empowerment comunitario.

\begin{abstract}
The community intervention from Social Work to provide answers to the needs, problems and aspirations throughout with its challenges and opportunities, and from a strategic and participatory approach in accordance with a theoretical-conceptual and methodological consistent. The article presents the methodological process linked to social work with communities focused on local development model strategic, comprehensive and sustainable structured around processes characterization and liaison with community strategic analysis, formulation of intervention, intervention implementation and evaluation proposal and / or reformulation of the intervention model. In each of the processes detailed methodology, objectives, techniques and professional skills and competencies required. To end describes the fields of community social work response to social
\end{abstract}

\footnotetext{
* Doutor e licenciado em Sociologia pela Universidad de Murcia. Graduado em Serviço Social pela Universidade de Alicante. Professor titular da Universidad de Murcia. Diretor da Facultad de Trabajo Social da Universidad de Murcia. Líder do grupo de investigação "Trabajo Social y Servicios Sociales". Múrcia, Múrcia, Espanha. E-mail: epastor@um.es
} 
protection systems, describing objectives, equipment and actions to be taken in social work with communities in Spain.

Keywords: Social work communities. Social change. Community empowerment.

Recebido em: 01/05/2013. Aceito em: 24/05/2013.

\section{Fundamentos teóricos que sustentan y orientan el proceso metodológico: "la brújula"}

Precisar las bases sobre las que se interviene favorece la construcción y validación de modelos teóricos desde la misma práctica, aspecto fundamental en la construcción de toda disciplina, desde una perspectiva crítica y dialéctica con la realidad institucional y política (REID, 2003, PASTOR, 2010a). Se considera necesario que el proceso metodológico integre los diferentes enfoques teóricos y metodológicos, adaptando estos a la idiosincrasia del contexto, momentos/procesos de análisis/intervención y sentido/resultados de la acción profesional. En este sentido, la teoría ecosistémica (teoría de sistemas, ecológica y redes sociales), la integración de las aportaciones del enfoque de potenciación, defensa, influencia social y poder nos proporcionan un marco conceptual de primer orden.

Actualmente se están desarrollando fundamentalmente dos perspectivas como base teórica para el trabajo social con comunidades. La primera, basada en los sistemas sociales, trata sobre las interacciones funcionales de las organizaciones, las burocracias y las comunidades, con especial énfasis en las distintas funciones de los sistemas - la adaptación y el crecimiento, el logro de objetivos, el mantenimiento del sistema y la integración/ cohesión. El segundo enfoque se basa en una perspectiva ecológica que nos permite una visión holística e integradora de la comunidad.

Vinculado a esta última, la teoría de redes sociales nos proporciona el marco apropiado para realizar un análisis e intervención comunitaria integradora de los diferentes elementos y relaciones, existentes o potenciales, existentes en una comunidad. Esta teoría es un encuadre ecosistémico que nos introduce en la estrategia de intervención comunitaria en redes sociales, basado en su identificación, análisis e intervención dinámica en red en el ámbito local. Vinculados a este marco teórico más amplio, destacamos un marco teóricoconceptual operativo de corto alcance que permite enmarcar la intervención comunitaria, concretamente la teoría no directiva (BATTEN, 1969; ROGERS, 1989; TWELVETRES, 1976, 1988, 1996; ROBERTIS; PASCAL, 1994; FREIRE, 1972, 1997a, 1997 b) etc.), la teoría de la motivación, necesidades (HELLER, 1996) y capacidades (SEN, 1987, 2000; MAX-NEEF, 1994; NUSSBAUM, 2002, 2012), desarrollo humano sostenible (MAX-NEFF, 2007, CLARK, 2000, PASTOR, 2011a, CITOLIN; ALFONSO, 2012), la dinámica de grupos y el conflicto como elemento clave del cambio (GALTUNG, 1995, GALTUNG; JACOBSEN, 2000; PASTOR, 2012 , entre otros). La teoría no directiva y de las capacidades humanas permite concebir a los grupos y organizaciones con capacidades y habilidades, precisando descubrirlas para que éstos sean sujetos activos de su propia realidad, es decir, sean competentes para construir ciudades y políticas competentes. Desde esta perspectiva, la intervención se orienta a generar contextos adecuados que permitan a los grupos y organizaciones disponer de oportunidades para (re)descubrir y desarrollar esas capacidades y habilidades.

La intervención comunitaria basada desde estos fundamentos teóricos se orienta a satisfacer las necesidades sentidas, convirtiéndose éstas en verdaderos "motores" de la sensibilización/ concienciación individual, grupal y comunitaria y su transferencia a una acción colectiva. Un trabajo social con comunidades centrado en los procesos y en las tareas de los grupos y las organizaciones comunitarias se oreinta en dos direcciones:

a) consecución de una adecuada cohesión interna (proceso), contribuyendo a que los grupos y las organizaciones encuentren cauces de conciliación entre objetivos y necesidades individuales y grupales, mediante estrategias

144 Emancipação, Ponta Grossa, 13(1): 143-158, 2013. Disponível em <http://www.revistas2.uepg.br/index.php/emancipacao> 
de dinámica de grupos - intragrupales -, por tanto, orientado a la construcción de contextos competentes, a recuperar confianzas, desarrollar la innovación, crear solidaridades, generar capacidades y liderazgos endógenos - una red de desarrollo local;

b) realización de acciones dentro de un marco temporal determinado, orientado a la consecución de objetivos (tareas), apoyando a los grupos y organizaciones para conseguir una productiva proyección externa, mediante estrategias intergrupales y de influencia en procesos políticos tangibles (PASTOR, 2010a, 2010b).

La planificación se basa en un marco teórico conceptual que proporciona un modelo de intervención comunitaria orientado a transferir los conocimientos formales a las personas, grupos y organizaciones comunitarias para que éstas adquieran las capacidades y habilidades necesarias para:

- identificar las causas del malestar o hechos detonantes;

- definir las necesidades, atribuyendo significado a las causas de las dificultades;

- buscar soluciones a sus problemas;

- valorar y elegir la más adecuada;

- determinar cómo se organizará la acción;

- ejecutar lo planeado;

- evaluar el grado de satisfacción respecto a los objetivos alcanzados, e

- innovar acciones futuras desde el aprendizaje deliberativo de lo realizado.

\section{El empowerment comunitario}

Se propone una metodología que apuesta por el cambio social sostenible a través de la investigación-acción participativa de los actores implicados, en particular, y de la ciudadanía, en general. Metodológicamente la acción participativa es un proceso circular y complejo en el que de forma espiral y continua, en cada una de las fases, se desarrolla un proceso secuencial, deliberativo y circular de planificación/acción/obtención de información sobre la acción desarrollada. La comunidad, grupos y organizaciones pasan a ser sujetos activos y reflexivos-creativos de la investigación, planificación y acción social.
Para el adecuado desarrollo de este proceso es necesario partir del estudio de las necesidades sentidas por los miembros de la comunidad y de una continúa y sincera comunicación entre/con y para los sujetos (ciudadanos y sus representaciones). El estudio requiere enfatizar prácticas y técnicas cualitativas que permitan el conocimiento de la realidad social y su transformación, poniendo el acento en la participación en todo el proceso y en el estilo del profesional en su quehacer cotidiano, de una manera sustantiva para provocar un desarrollo verdadero, humano y sostenible. El concepto operativo del empowerment resume $\mathrm{y}$, a la vez, complejiza un modelo de intervención comunitaria basado en los procesos de capacitación y transferencia de conocimientos, capacidades y habilidades a la población, desde una perspectiva crítica (FALEIROS, 2003) y dialéctica.

El proceso de intervención comunitaria recurre a una voluntad y compromiso de concertación y a la creación y fortalecimiento de partenariados y redes sólidas y sostenibles. Para ello, es fundamental adoptar posiciones claras y positivas del conflicto y de la negociación para su inclusión transversal. El tratamiento adecuado de los conflictos comunitarios con las autoridades permite el desarrollo y crecimiento de las redes comunitarias. La práctica comunitaria requiere comprender que no todas las relaciones se basan en un objetivo e interés compartido. Con frecuencia y normalidad, los grupos tienen objetivos opuestos o distintos y no responderán, únicamente, mediante destrezas, tales como la empatía. El/la trabajador/a social en la intervención colectiva adopta múltiples roles para adaptarse a los ritmos y rostros: defensor, mediador, coordinador, planificador, motivador etc. Todo ello representa la versatilidad necesaria para interactuar con una comunidad compleja, en la que el trabajo social debe "equiparse" de un discurso integrado e integrador de las características y dinámicas de la población, grupos y momentos, de manera que, con técnicas y habilidades innovadoras, puedan contribuir a construir caminos que, con la lógica participativa, se encuentran sin prescribir (¿qué?) y que requieren de sus actores ser competentes en el caos del complejo universo relacional.

La práctica comunitaria es un modelo de intervención orientado a transferir los 
conocimientos formales a las personas, grupos y organizaciones comunitarias para que estas adquieran las capacidades y habilidades necesarias para: identificar las causas del malestar o hechos detonantes, definir las necesidades, es decir atribuir significado a las causas de las dificultades, buscar soluciones a sus problemas, valorar y elegir la más adecuada, determinar cómo se organizará la acción, ejecutar lo planeado y evaluar el grado de satisfacción respecto a los objetivos alcanzados e innovar acciones futuras.

En este proceso el sujeto desarrolla el pensamiento lógico, "aflorando" sus capacidades, a la vez que proporciona al profesional el conocimiento informal que éste precisa. Esta retroalimentación circular e infinita entre profesional-sujetos con los que realizamos el "juego democrático" del cambio social, conduce a un auténtico análisis e intervención comunitaria, en el que las relaciones entre trabajo social y grupos/organizaciones comunitarias se caracterizan por la interdependencia y cooperación, promoviendo sujetos activos de su propia realidad que saben lo que desean y se sienten capaces de hacerlo, ya que el sujeto social "dispone de relaciones que son fuerzas para tornarse protagonista, con la elaboración y articulación de sus relaciones en el cotidiano, articuladas al soporte social, al cuidado en tanto derecho" (FALEIROS, 2011, p.125).

\section{Una metodología para impulsar cambios sociales sostenibles y autónomos en el complejo universo relacional}

El proceso metodológico se plantea a partir de objetivos centrados en la tarea y en el proceso. Los primeros están orientados hacia la consecución de tareas con resultados tangibles y los segundos se vinculan a la creación e impulso de estructuras y dinámicas comunitarias que permitan su autodeterminación. En este caso le hemos denominado "grupo/red motor del desarrollo local" - en el que el/a trabajador/a social está siempre detrás de la cabeza del grupo o equipo de organización comunitaria.

\section{Caracterización y enlace con la comunidad}

Se propone un proceso de investigaciónacción sistemático, creativo/innovador y reflexivo/deliberativo que incorpora las diversas perspectivas que configuran la realidad social, relacional e institucional de una determinada comunidad y proporciona los elementos suficientes para analizar e interpretar la realidad (diagnóstico), además de facilitar la toma de decisiones para el posterior diseño de la intervención (líneas posibles de intervención).

Se diseñan unas líneas estratégicas progresivas y flexibles de acercamiento a la realidad comunitaria en íntima relación con los actores sociales que interactúan y construyen la comunidad que deseamos conocer. Es un estudio basado en un conocimiento cuantitativo y cualitativo de la realidad de la comunidad, sus interrelaciones, tendencias, centros de interés, recursos existentes y potenciales, potencialidades y dificultades etc. El reto se centra en el diseño de estrategias que permitan la máxima diversidad en la audición, incorporando al proceso, la compleja red de actores e interrelaciones que coexisten en la comunidad.

El proceso de aproximación a las realidades comunitarias conlleva básicamente dos estrategias simultáneas:

a) exploración y descripción de la comunidad a través de un procedimiento indirecto de recogida de información e

b) identificación de las necesidades sentidas y de las redes relacionales existentes en el territorio, articuladas mediante un proceso concreto centrado en las manifestaciones de sus actores respecto de ésta.

En un primer momento, se elaborará un perfil de la comunidad en torno a la información más externa (procedimiento indirecto), objetiva (haré) cuantificable y cuantificada. Para ello se utilizarán técnicas de conocimiento como la observación y el análisis documental, tanto de fuentes internas de los servicios (informes, demandas registradas, evaluación, programas y proyectos realizados) como externas. Simultáneamente, se desarrollan las entrevistas informales o contactos, lo que favorecerá la progresiva aproximación a la información subjetiva (sofá), basada en el discurso de profesionales, personas clave, 
líderes y ciudadanos, mediante técnicas más cualitativas. Ambas actuaciones se enriquecen/ retroalimentan mutuamente, dado que el primero nos permite identificar a actores y redes sociales; a la vez el contacto con éstos nos permitirá identificar las necesidades sentidas y focalizar progresivamente la investigación-acción.

Impulsar un trabajo social en perspectiva comunitaria supone organizar el servicio y el trabajo cotidiano de manera muy diferente al "tradicional". En muchas ocasiones, nos encontraremos con obstáculos institucionales y poblaciones para estructurar el servicio y la actividad profesional con una perspectiva comunitaria. Los responsables institucionales, los grupos, las organizaciones y las personas han adquirido, de manera general, una imagen del servicio y de nuestra actividad profesional más cercana a lo individual, a lo paliativo, a lo asistencial etc. Con el paso del tiempo se ha construido una representación social de nuestro rol, cometidos y objetivos no acordes, en la mayoría de las ocasiones, a un trabajo con dimensión comunitaria y, por ello, cuando pretendemos dar un giro a este estilo profesional debemos de re-construir de "nuevo" esta imagen, estas expectativas etc., desde la "palabra", pero también desde la "acción".

Se persigue identificar los centros de interés, oportunidades y situaciones de dificultad que puedan convertirse en punto de partida de análisis e intervención comunitaria. Para ello es necesario que sean percibidas/sentidas como importantes y vitales por los propios interesados, en un doble sentido:

a) provocar insatisfacción o malestar en la población (personas, grupos y organizaciones) y

b) ser capaces de movilizar a personas y grupos a encontrar soluciones colectivas y provocar cambios en la realidad comunitaria.

La investigación-acción se inicia con un proceso constante de contacto con el territorio y sus habitantes, en un doble sentido: objetivo e intersubjetivo que conoceremos mediante la aproximación directa a los espacios públicos y privados. De esta manera podemos identificar dos objetivos generales, por una parte, los de conocimiento (tarea) y, por otra, los de proceso. Respecto de los primeros nos interesa conocer: las necesidades sentidas de la población, cómo las prioriza ella misma, las soluciones que considera para resolver las necesidades, a quienes responsabiliza de las soluciones y a qué se compromete para resolver esos problemas. En relación a los objetivos de proceso nos referimos a: iniciar la toma de conciencia individual y colectiva acerca de las necesidades identificadas y compartidas, incorporar a las personas al proceso de participación, desarrollar capacidades y habilidades para que definan y prioricen necesidades $y$, ofrecer nuevos patrones de interrelación colectiva.

En la estrategia de aproximación, es necesario cuidar el proceso de captación y acercamiento de/a los informantes, siendo recomendable realizarlo a través de las propias redes sociales que configuran su contexto natural, dado que favorecerá la interacción conversacional y la "semilla" para crear el grupo/ red de desarrollo local, que asumirá el proceso.

Este grupo/red de desarrollo local supone un reconocimiento y un compromiso de todos aquellos que pueden y desean contribuir eficazmente al proyecto de acción y transformación de la comunidad. Un grupo/red diverso (incorpora personas, grupos y entidades dispuestas a colaborar en diferente grado), inclusivo (abierto a la incorporación de nuevos actores en el proceso), sostenible (autogestionada por la misma red), flexible y abierto (estructurada en torno a una red vinculada entre si, utilizando el grupo representativo y su conexión a las organizaciones y grupos). Este grupo/red motor (Consejo Ciudadano y Comités de Tarea) se iniciará con su presentación institucional y pública - que reconozca la labor a desarrollar por las personas y entidades integrantes y el compromiso de las autoridades locales para su impulso y apoyo. Se constituirá el Consejo Ciudadano, u otro mecanismo de participación que permita la organización coordinada de la comunidad para la acción colectiva, sobre la articulación en torno a comités-comisiones en función de intereses y necesidades. La comunidad debe sentir que es ella y no los profesionales, quien asume las responsabilidades. 


\section{Análisis Estratégico}

Supone un proceso que implica identificar y conceptualizar las necesidades sentidas por la comunidad, sus causas y evolución a lo largo del tiempo, así como los factores condicionantes y de riesgo, sus tendencias previsibles y las potencialidades de desarrollo. Es un proceso que permite la jerarquización de las necesidades y el establecimiento de prioridades y estrategias de intervención, de manera que pueda determinarse con anterioridad a la elaboración de planes, programas y proyectos, los grados de viabilidad y factibilidad de los mismos, atendiendo a los medios disponibles, las fuerzas y actores sociales involucrados, así como los obstáculos previsibles con los que nos podemos encontrar en el camino. Ello requiere dos estrategias concatenadas: la primera, orientada a la jerarquización de los problemas que nos permita focalizar y construir los escenarios de intervención y, la segunda, destinada a analizar la viabilidad de las alternativas de intervención antes de la programación.

Analizar la viabilidad de las estrategias con el grupo/red motor, supone revisar si las alternativas seleccionadas son viables en la práctica, valorar la efectividad de que una idea sea llevada a la práctica, considerando los obstáculos presentes y potenciales. En definitiva, supone un proceso participativo de análisis, reflexión, discusión y toma de decisiones.

Pero esta "fase" tiene como objetivo de proceso el consolidar el grupo motor, de manera que favorezca la emergencia de lideres endógenos, permita que los miembros "airen" sus diferencias y reconozcan el papel de protagonistas, creen comités o redes específicas que fomenten el compromiso, mantengan el interés, establezcan marcos temporales y aporten retroalimentación etc. Es el momento de la "fusión" entre el grupo motor y el resto de grupos e instituciones locales.

\section{Formulación y formalización de la intervención}

El objetivo es diseñar y formalizar con el grupo/red motor el Plan, Programas y/o Proyectos de gestión e intervención comunitaria integrados en clave estratégica. Se trata de articular y acordar los fines y objetivos a los que se dirige la intervención en un proceso en el cual se "seleccionan, ordenan y diseñan las acciones que deben realizarse para el logro de determinados propósitos, procurando una utilización racional de los recursos disponibles" (SUAREZ; DIÉGUEZ, 2002, p.84). El/a trabajador/a social formalizará conjuntamente con los sujetos sociales la intervención a realizar, a partir de la operatividad del grupo/red.

Las condiciones para elaborar una adecuada planificación podemos sintetizarlos en:

a) objetivos posibles, medibles, específicos, concretados en acciones, temporalizados y con responsables de ejecución;

b) medios y recursos disponibles y eficaces;

c) acciones integrales y transversales;

d) precisión en el sistema de evaluación e indicadores que permitan retroalimentar la investigación-acción y adoptar decisiones;

e) favorecimiento de la participación; y

f) mejoraramiento del bienestar y los procesos de desarrollo local.

Es fundamental que el diseño de la planificación sea formalizada por el grupo/red, mediante compromisos y acuerdos programáticos que establecen mecanismos de seguimiento y evaluación de responsabilidades compartidas, acciones previstas y realizadas, así como de los resultados obtenidos. Pero la programación, más allá del objetivo centrado en la tarea, persigue capacitar a los lideres, grupos y organizaciones locales para que puedan promover cambios de forma autónoma y resolver por si mismos las situaciones que surjan. El grupo motor debe percibir que no se trata de establecer un "contrato" con el profesional o con una determinada institución, sino con ella misma y la comunidad a la que pretende servir. Se trata, por tanto, de "compromisos-contratos-acuerdos" entre los grupos y redes locales, donde el profesional se sitúa en un rol de asesoramiento técnico cuando este es preciso.

En este sentido, es fundamental que el grupo/red de desarrollo se estructure y organice en torno a redes centradas en la tarea, siendo liderados por miembros pertenecientes al propio grupo motor (liderazgo endógeno). El grupo/ red de desarrollo debe liderar la presentación

148 Emancipação, Ponta Grossa, 13(1): 143-158, 2013. Disponível em <http://www.revistas2.uepg.br/index.php/emancipacao> 
pública de los proyectos a emprender, con una doble finalidad, legitimar y protagonizar al propio grupo/red y a los subgrupos, y a la vez comprometer a sus miembros ante la comunidad. Por su parte, la comunidad, se convierte en sujeto de intervención al conocer de antemano las acciones a emprender y disponer de canales de participación en las mismas. No se trata de "preparar a la comunidad", como usualmente se señala, sino de involucrarla en el proceso.

\section{Implementación de la propuesta de intervención comunitaria}

El objetivo es impulsar, fortalecer y consolidar procesos y cauces de participación ciudadana, organización comunitaria, coordinación interinstitucional y gestión e intervención colectiva de las situaciones de dificultad. Se trata de poner en marcha las estrategias, los programas y/o proyectos planteados con sus "gentes", fortalecer la identidad y sentido colectivo de la comunidad, consolidar el grupo/red de desarrollo organizando y consolidando grupos productivos y lideres endógenos mediante procesos de autocapacitación. La intervención comunitaria debe caracterizarse, a su vez, por influir en los procesos y en las políticas, de manera a promocionar un modelo de desarrollo sostenido y duradero, orientado a introducir cambios en los procesos sociales, desde un punto de vista tanto preventivo como de inserción social, y a la vez influir en decisiones institucionales y políticas mediante acciones de organización de la comunidad y coordinación de esfuerzos y recursos.

Es un nivel de "acompañamiento" supervisión y coordinación de grupos y procesos, evitando duplicidades, activismos, prácticas puntuales e inconexas etc. Las actividades a emprender en esta fase serán tan diversas como sean los proyectos que se vayan a implantar. El profesional social trabajará con los subgrupos y redes, así como con el grupo/red motor, proporcionandoles el apoyo técnico para asegurar el componente técnico-científico del trabajo que se realiza respecto a organización, planificación y evaluación. Desempeñará múltiples actuaciones, entre ellas: entrevistas, encuentros, asambleas comunitarias - sectoriales o zonales -, talleres comunitarios de sensibilización, movilización y solidaridad; acturá en la formación de lideres, mediación comunitaria e intercultural, en la organización y dinamización de las formas organizativas colectivas (comisiones, comités, redes temáticas etc.) y en la creación de grupos de ayuda mutua y autoayuda (poner en contacto las personas entre si, ayudar a los que ayudan, generar contactos etc.). Además, gestionará la concertación y administración de recursos, sensibilización y divulgación en medios de comunicación local, seguimiento y supervisión de grupos y redes, evaluación de las formas organizativas y sus resultados, formalización y consolidación grupos productivos etc.

\section{Evaluación, validación y/o reformulación del modelo de intervención}

El objetivo es evaluar la intervención comunitaria y elaborar un modelo de gestión e intervención comunitaria sostenible y duradero, a partir del escenario inicial y el logrado. La evaluación de la práctica comunitaria es un proceso continuo que se encuentra presente en todo el proceso - al finalizarla evaluamos el proceso y resultados obtenidos con el fin de aprender de la práctica y transferir, nuevamente, ese conocimiento a la comunidad local y científica.

La evaluación tiene que ser: útil, práctica, realista, participativa, creativa y objetiva. Los indicadores tienen que presentar constantes tales como:

- pertinencia, es decir reflejar la situación o el fenómeno que van a mediar;

- adaptabilidad - utilización diversa de datos y fuentes $y$

- sencillez - comprensible para todos los actores con independencia de su cualificación.

Por tanto, el proceso de evaluación debe ser lo más sencillo, flexible y ligero posible, evitando las operaciones que sólo sirven para medir los procedimientos y los programas y dejan en segundo plano los objetivos de proceso, ya que la evaluación tiene objetivos de resultados (tareas) y de proceso.

Es importante realizar una evaluación de las transacciones del grupo/red de desarrollo entre si y con el entorno. Se evaluará la evolución y cambios de los objetivos de los grupos productivos, la naturaleza de los lazos creados por la red entre cada una de las instituciones 
entre si y con otras, las alianzas y las redes de cooperación y solidaridad que han emergido con la intervención comunitaria etc.

En el proceso de intervención aparecen obstáculos que pueden dificultar el logro de los cambios previstos en el entorno. Es importante revisar completamente los objetivos y las actuaciones, una vez que se evaluará por qué las actividades no han alcanzado los propósitos planteados. El cuestionamiento debe contribuir a que el grupo/red valore fracasos y éxitos y aprenda de ellos.

Podemos distinguir dos tipos de evaluación: interna y externa. En la interna es imprescindible que participen los actores sociales involucrados en el proceso, dado que de esta manera atenderemos a objetivos de tarea (evaluar las acciones emprendidas por cada grupo y red productiva y por el mismo grupo motor) y de proceso (reconocer las posibilidades de cambio local, liderar nuevos procesos etc.). Es aconsejable, en ocasiones, realizar una evaluación externa, tanto desde la opinión de expertos ajenos al proceso como de los ciudadanos locales. La evaluación interna y externa no deben reflejarse en meros "Informes Evaluadores", sino en la configuración de un proceso socioeducativo de retroalimentación de ambas evaluaciones y entre actores, por medio de reflexión y debate/deliberación grupal sobre lo realizado y las acciones siguientes y la validación de un modelo de intervención construido a partir de prácticas comunitarias (auto-evaluación).

Es fundamental, de nuevo, reconocer y recompensar los esfuerzos y las actuaciones emprendidas por las personas y entidades involucradas, fortaleciendo así el sentimiento de la comunidad e incentivando la emergencia de nuevos proyectos y líderes. El éxito logrado, por pequeño que sea, proporciona un sentido de realización, una nueva percepción del problema y una confianza renovada en su capacidad para manejar problemas futuros. El reconocimiento tiene el propósito de producir sentimientos positivos de logro. El grupo/red motor de desarrollo local protagonizará, de nuevo, la presentación del "producto" del proceso: el Informe Final de Resultados.

\section{Ámbitos de intervención comunitaria en Trabajo Social en España}

Los ámbitos profesionales son los espacios delimitados por la organización institucional de los servicios en los que opera el trabajador social y por el marco programático y operativo en el que se concreta y desarrolla su labor (ANECA, 2005). En su elaboración se han considerado las diferentes normativas vinculadas a los sistemas: el Libro Blanco del Titulo de Grado en Trabajo Social (ANECA, 2005), el Código Deontológico de Trabajo Social (CONSEJO GENERAL DE TRABAJO SOCIAL, 2012), prácticas comunitarias en distintas comunidades autónomas y autores (SAAVEDRA, 2004; RUIDIAZ, 2004; NATÓ et. al., 2005; MALAGÓN, 2008; PUYOL, 2009; COLOM, 2010; FERNÁNDEZ; LÓPEZ, 2008; HOLGADO; MAYA, 2012; LÓPEZ, 2012; PASTOR, 2012, PASTOR; HUERAS, 2012, entre otros).

\section{El trabajo social con comunidades en el sistema de servicios sociales}

Es el ámbito más habitual y donde se concentran la mayoría de las experiencias de intervención comunitaria consideradas "puras", tanto desde la atención primaria como especializada. La atención primaria, general, comunitaria o de base constituye el cauce normal de acceso al sistema de servicios sociales y prestan una atención integrada y polivalente en el ámbito más próximo al ciudadano y a su entorno familiar y social, teniendo su justificación última precisamente en la atención comunitaria. En este sentido, entre sus objetivos principales se encuentran el reforzamiento de la comunidad local y la potenciación del apoyo social del individuo/familia/comunidad. Por otra parte, los servicios sociales especializados se dirigen a aquellas personas o colectivos que, por sus condiciones de edad, sexo, discapacidad, u otras circunstancias, precisan de especial protección social por medio de recursos o programas específicos. En este último nivel, la intervención comunitaria se centra más en fomentar al asociacionismo y el tejido social y se interviene principalmente a través del denominado "tercer sector".

Partimos de considerar que el objetivo general del Trabajo Social con comunidades en 
el sistema de servicios sociales es el impulso de la acción colectiva para satisfacer necesidades/ problemas identificadas y detectadas por ellos mismos. Las principales actuaciones que desarrolla el/la trabajador/a social son:

- Realización de investigaciones básicas de aspectos epistemológicos de la disciplina y divulgación científica de las experiencias.

- Formulación de planes, proyectos, y programas dirigidos a solucionar una carencia detectada en el diagnóstico comunitario participativo.

- Prevención y detección de situaciones emergentes que puedan incidir negativamente en un territorio y por ende en su comunidad teniendo como finalidad una actuación precoz sobre las causas.

- Evaluación de resultados en relación a objetivos identificados, teniendo en cuenta técnicas, medios y tiempo empleado y como finalidad, la viabilidad de las intervenciones comunitarias.

- Capacitación a la comunidad para actuar de manera organizada, constituyendo una comunidad de intereses y de acción, para que sea ella misma capaz de responder a las necesidades que presentan con la utilización de sus recursos propios existentes y potenciales.

- Promoción y dinamización de la participación comunitaria, el asociacionismo, la ayuda mutua etc, en definitiva, impulsa la implicación de la ciudadanía en la puesta en marcha de iniciativas comunitarias fortaleciendo la cohesión social.

- Mediación en conflictos con el fin de unir las partes implicadas y posibilitar que sean los propios interesados quienes logren la resolución del problema, dentro de la comunidad, o entre éste y agentes o instituciones externos.

- Participación y apoyo en la incorporación de las políticas sociales de forma transversal en las actuaciones generales de la administración correspondiente, abogando por servicios, perspectivas y políticas sociales justas que den respuestas a situaciones de desigualdad social.

- Articulación del trabajo en red y en coordinación interinstitucional e intrainstitucional de cara a dar respuestas integrales a las necesidades complejas.

En cuanto a los equipamientos donde se desarrolla el Trabajo Social con comunidades, se puede identificar como fundamentales los Centros de Servicios Sociales (Centros de Acción Social, Centros Sociales Polivalente) y Centros de Acción Comunitaria (Centros municipales de barrios, o de base, o sociales o comunitarios). Los primeros son equipamientos generalistas/ polivalentes que ofrecen servicios y recursos destinados a toda la población. Respecto a los Centros de Acción Comunitaria, se tratan de equipamientos específicos en la intervención comunitaria u espacios de participación y colaboración social del y para el barrio, donde se ofrece una atención a los colectivos desde su propio entorno convivencial.

Además de los equipamientos enunciados anteriormente es posible desarrollar acciones comunitarias en un nivel especializado en albergues, centros de estancias diurnas, centros de conciliación de la vida familiar y laboral, centros de acogida etc.

Para el desarrollo de la intervención comunitaria dentro del sistema resulta imprescindible desarrollar un trabajo sistemático de colaboración y coordinación de las diversas instituciones y entidades de los ámbitos escolar, familiar, social, político etc. Se trata, por lo tanto, de utilizar y beneficiarse de todos los recursos y equipamientos sociales existentes (centros sociales y culturales, equipamientos deportivos, educativos y religiosos, asociaciones y grupos informales, centros privados que presten servicios sociales etc.) para intervenir de manera conjunta e integral abarcando la solución a necesidades globales que se plantean en el Trabajo Social con comunidades. Esto conlleva una articulación comunitaria horizontal, en red y por medio de mecanismos y protocolos estables de colaboración entre los diferentes ámbitos de atención.

\section{El trabajo social con comunidades en el sistema de salud}

Básicamente la línea de intervención que define el Trabajo Social en este ámbito es la prevención, educación y promoción de la salud, sin olvidar el fomento de la red social de apoyo. De ahí, que las principales actuaciones de intervención del trabajo social con comunidades en este ámbito se centren en: 
- Estudio de los factores psicosociales con incidencia en la salud y que favorecen o dificultan la implementación de los programas de salud; análisis de los procesos sociales emergentes y sus posibles consecuencias respecto de la salud de la población; actitudes y comportamientos de los grupos sociales respecto de la salud y la enfermedad (demanda y utilización real de los servicios sanitarios; niveles de adecuación de los servicios existentes, según los principios de eficacia, eficiencia y calidad etc.).

- Diseño e implementación de programas preventivos orientados a identificar de manera precoz la población de riesgo de necesitar apoyos y ayuda.

- Creación de grupos de ayuda mutua, voluntariado, asociaciones de familiares con diagnósticos médicos específicos (diabetes, insuficiencia renal etc.), teniendo en cuenta las necesidades de la población de la Zona Básica de Salud y apoyando junto al resto del equipo en aquellas actividades que necesiten la actuación de profesionales de la salud (charlas, educación sanitaria etc.).

- Promoción de redes de colaboración entre los Equipos de Atención Primaria y el tejido social de la Zona Básica de Salud.

- Mediación en los conflictos del paciente con su entorno y en relación al proceso (relación con el sistema sanitario, acuerdos familiares, organización de cuidados etc.).

- Fomento de una cultura de educación de la salud comunitaria con la participación de pacientes, familiares y personal sanitario.

Los profesionales del trabajo social se adscriben a equipamientos sanitarios públicos (centros de salud, hospitales, centros de rehabilitación psicosocial y laboral, centro de atención a drogodependientes, centros de salud mental etc.), centros residenciales, asociaciones, mutuas y empresas privadas que presten servicios de sanidad.

La diversidad de disciplinas profesionales en el ámbito sanitario (médicos, psicólogos, enfermeros y trabajadores sociales) favorece una intervención integral pero también aporta una mayor complejidad, lo que puede afectar su funcionamiento. Por ello, es necesaria una distribución adecuada de los roles, de manera que permita la complementariedad de funciones junto al intercambio de recursos (HOLGADO;
MAYA; PALACIO, 2012). En este sentido, cobra mayor fuerza la necesidad de coordinación tanto intrainstitucional con otros servicios del sistema (Salud Mental, Salud Laboral, Inspección etc.) como interinstitucional y entre profesionales.

\section{El trabajo social con comunidades en el sistema educativo}

El trabajo social comunitario encuentra su justificación en la propia Ley Orgánica de Educación $n^{\circ} 2$, de 3 de Mayo de 2006, al señalar como uno de sus principios fundamentales la calidad y la equidad y al destacar la atención a la diversidad como un principio no sólo como una medida.

En la actualidad la figura del trabajo social ejerce sus funciones tanto en la educación formal (Equipos de Orientación Psicopedagógica, Departamentos de Orientación, educación especial) como no formal (actividades formativas para determinados sectores de población). Sus acciones, fundamentalmente, se dirigen a intervenir "dentro" de los centros escolares, aunque cada vez es mayor la necesidad de incidir en las relaciones de los centros educativos con las redes y recursos sociales de la zona donde se ubica (apoyo social, asociaciones, servicios sociales, sanidad etc.), para de esta forma ofrecer respuestas integrales a las necesidades/ demandas actuales que requieren el sistema educativo, las personas usuarias y la propia comunidad.

En líneas generales el trabajador social con comunidades en el ámbito educativo tiene como funciones principales la prevención y asistencia en temas como el absentismo y el fracaso educativo, la integración de inmigrantes y colectivos desfavorecidos, la detección de malos tratos y abusos sexuales, la mejora del clima de convivencia, la atención ante el fenómeno de la violencia, o la estimulación de la mejora pedagógica o la participación de todos en la buena práctica de los procesos (HERNANDEZ; GONZÁLEZ; CIVICOS; PEREZ, 2006). Las actuaciones de la intervención del trabajo social comunitario en el sistema educativo son:

- Estudio sobre la influencia de los factores en el propio sistema educativo y del impacto de este último sobre el sistema social, además de investigar causas del absentismo y fracaso 
escolar, integración de inmigrantes y colectivos desfavorecidos, mejora del clima de convivencia escolar etc., en colaboración con otros servicios externos e institucionales.

- Participación en la elaboración y desarrollo de programas de prevención y detección de situaciones-problemas dirigidos a la comunidad educativa, teniendo como fin evitar procesos de inadaptación escolar, absentismo, fracaso escolar etc.

- Programación de actuaciones comunitarias en colaboración con instituciones, asociaciones, agentes sociales de la zona etc.

- Fomento y apoyo del asociacionismo en el ámbito escolar como proceso de aprendizaje para inculcar valores de participación e implicación en la resolución de las problemáticas comunitarias.

- Establecer relaciones de cooperación con otros profesionales para el diseño y ejecución de las políticas sociales en materia de educación de acuerdo con las características sociológicas y de las necesidades del entorno.

Respecto a los equipamientos donde se llevan a cabo estos objetivos podemos mencionar los Centros de Atención Infantil y Primaria (CEIP), Centros de Educación Segundaria (IES), Centros de Educación Especial, Centro de Educación de Adultos, entre otros. La coordinación es un aspecto fundamental de su trabajo manteniendo líneas de coordinación y cooperación tanto con profesionales de otros ámbitos públicos y privados (servicios sociales, sanidad, asociaciones etc.) como con los profesionales del ámbito educativo (profesores, director/a, jefe/a de estudios etc.).

\section{El trabajo social con comunidades en el ámbito de la justicia}

El ámbito judicial-penitenciario es un campo en el cual el Trabajo Social con comunidades está relacionado, principalmente, con la gestión de las penas alternativas de arresto de fin de semana y trabajo en beneficio de la comunidad, así como la participación en las medidas de seguridad privativas de libertad y el seguimiento de las penas referidas y de las medidas que procede por parte de los servicios sociales penitenciarios. La posibilidad de llevar a cabo intervenciones comunitarias desde el Trabajo Social es muy limitada, siendo indispensable realizarlas desde la comunidad "antes" del delito, llevándose a cabo desde una perspectiva de prevención. Las prácticas comunitarias se orientan, fundamentalmente, a la prevención terciaria desde una perspectiva psicosocial. Las principales actuaciones del trabajador social son:

- Investigación para la elaboración de programas de seguimiento de medidas en medio abierto, alternativas a prisión, inserción sociolaboral, programas de prevención etc.

- Colaboración, cuando se le requiera, en el Consejo Social Penitenciario, teniendo entre sus objetivos dar mayor protagonismo a las organizaciones del tercer sector y sensibilizar a la sociedad en los ámbitos de inserción laboral.

-Fomento de la participación de asociaciones y personas voluntarias en la dinámica de la programación de los talleres y actividades que se llevan a cabo en los centros penitenciarios.

- Potenciación de la labor externa de los servicios sociales favoreciendo el acceso de las personas usuarias externas de estos servicios fuera del centro penitenciario, dando así respuesta a los nuevos cometidos del Código Penal en materia de penas alternativas y medidas.

- Promoción de proyectos de mediación.

- Impulso de proyectos de trabajo en beneficio de la comunidad como una medida alternativa al ingreso en prisión fomentando la reeducación.

- Colaboración y participación con entidades para conseguir la reinserción y reeducación de las personas condenadas.

- Difusión de buenas prácticas con la participación del tercer sector que destaquen por su eficacia y originalidad y que sean susceptibles de generalizarse en otros establecimientos reconocer el alto grado de compromiso.

En el ambito judicial, el profesional social interactua con los siguientes equipamientos: Centros penitenciarios, CIS, Juzgados, Unidades de madres, comunidades terapéuticas extra-penitenciaria, unidades dependientes, módulos de respeto, asociaciones destinadas con el ámbito de justicia, justicia juvenil etc., aunque principalmente se desarrolla la intervención comunitaria desde los Servicios Penitenciarios Externos. 


\section{El trabajo social con comunidades en el ámbito de la vivienda-hábitat}

La vivienda es más que un espacio físico, es donde se cimientan muchos de los procesos de socialización. Actualmente nos encontramos con una situación plagada de necesidades/ problemas siendo la más significativa el aumento de los desahucios por el impago de las hipotecas debido a la situación económica del país. Por este motivo nuestro fin último es contribuir a la construcción de un discurso social sobre la vivienda y la lucha por un alojamiento digno. En relación a las actuaciones señalamos como fundamentales:

- Estudios sobre chabolismo, infravivienda, accesibilidad etc., para que los condicionamientos sociales sean una parte fundamental de las políticas de vivienda y para que se lleven a cabo intervenciones globales que incidan en los factores causales.

- Desarrollo de programas de dinamización comunitaria de barrios de nueva construcción o en proceso de remodelación, mejora del hábitat, de la vivienda, del barrio, planificación urbana etc.

- Participación en la formulación e implementación de los proyectos de reasentamientos poblacionales, procesos de promoción de vivienda con protección pública, planes de emancipación juvenil etc.

- Mediación vecinal cuando existan dificultades para llegar a acuerdos o conflictos, relaciones de convivencia muy deteriorados en el tiempo, comunidades multiculturales y de diversidad económica etc.

- Formación y capacitación de las personas que acceden a una vivienda normalizada, si muestran indicadores de vulnerabilidad o riesgo sobre el mantenimiento de viviendas y su encaje con el entorno peri doméstico y comunitario.

- Actuaciones de formación y capacitación para la adquisición de pautas de convivencia adecuadas, trabajando la participación, aceptación y compromiso en los procesos de realojo ya que, en determinadas comunidades, la adquisición de una vivienda no es el fin del proceso sino el principio, una vez que sus miembros están inmersos en unos procesos de exclusión transversal que no les permite el mantenimiento del hábitat en condiciones adecuadas. Debe existir una intervención con la comunidad antes, durante y después de los realojos en este sentido.

- Implicación en las plataformas reivindicativas de apoyo a las personas y familias en procesos de desahucio, influyendo en las políticas públicas.

En lo referente a las líneas de coordinación, cooperación, complementariedad resultan fundamentales la colaboración entre la Administración y las entidades sociales, no sólo porque los objetivos sociales sean los mismos, sino porque la coordinación, la complementariedad y la cooperación entre los agentes privados y públicos permiten aprovechar sinergias, compartir recursos y evitar la duplicidad de esfuerzos y recursos. La mejora del alcance y la eficacia de las intervenciones en materia de vivienda requiere la coexistencia coordinada de programas destinados a colectivos y realidades diferentes, impulsados tanto desde las Administraciones Públicas como desde las entidades sociales, de manera conjunta.

\section{La mediación comunitaria como ámbito de intervención}

Los dos grandes objetivos que se persiguen desde un servicio de mediación comunitaria son el fomento de la convivencia y la participación ciudadana. El horizonte de la acción mediadora es promover la convivencia, un vivir cotidiano entre personas que presentan distintos bagajes culturales en los cuales interaccionan entre sí, a partir de relaciones sociales e intercambios que desembocan en la construcción de una nueva cultura compartida, sin que esto implique la eliminación de las identidades de origen, aunque no se niegue su transformación y enriquecimiento. Desde el punto de vista comunitario, la convivencia persigue la prevención del conflicto $y$, en definitiva, la construcción de una sociedad participativa. Un elemento clave de la convivencia intercultural son las relaciones sociales e intercambios que tienen lugar de manera cotidiana en los barrios donde todos vivimos. Desde el servicio de mediación se colabora, junto a otros profesionales y entidades, para incentivar y potenciar ese tejido, ya que es uno de los canales por excelencia a través de los cuales tienen lugar esas relaciones sociales. Por

154 Emancipação, Ponta Grossa, 13(1): 143-158, 2013. Disponível em <http://www.revistas2.uepg.br/index.php/emancipacao> 
una parte, son un canal idóneo para conseguir que la población se involucre y se identifique con la vida social del distrito. Pero además, es desde el tejido asociativo que el barrio, el distrito, la comunidad de propietarios, las asociaciones de padres, los colegios, las organizaciones no gubernamentales etc., se abren a los nuevos vecinos.

Otro objetivo del servicio de mediación comunitaria se refiere al fomento de la participación social. La participación persigue favorecer, potenciar e incrementar la presencia de la población en los espacios sociales e institucionales del ámbito local. Con ello se contribuye a la construcción de una sociedad en que, a través de la participación, tiene lugar el establecimiento de nuevas relaciones sociales que facilitan la identidad grupal, la comunicación y crean cauces de prevención y manejo de los conflictos comunitarios. Las vías de participación pueden ser individuales o grupales. La participación individual consiste en la oferta de canales existentes para la integración de la persona en consejos escolares, asociaciones de vecinos, organizaciones no gubernamentales etc. La participación colectiva consiste en la promoción de la vida comunitaria local tanto de las asociaciones como de los grupos de personas que actúen como interlocutores y agentes sociales, desarrollando las relaciones y fomentando la creación de la comunidad y la potenciación de redes de ayuda mutua (PASTOR, 2009, 2012).

Los ámbitos de actuación del trabajo social en el contexto de la mediación comunitaria se agrupan en dos niveles de actuación: prevenciónsensibilización y gestión de los conflictos.

En cuanto a los programas de prevención y sensibilización, deben estar integrados en espacios de encuentro de todos los profesionales implicados en una temática concreta. El objetivo de los trabajadores sociales-mediadores es coordinarse con las actuaciones de los distintos profesionales a través de una acción integral para evitar duplicidad de funciones y solapamientos de esfuerzos de la población. Por la propia naturaleza del ámbito comunitario, los mediadores necesariamente han de trabajar en conexión con los demás profesionales a través del trabajo en red, así como con el resto de los profesionales que componen los Servicios
Públicos Locales y los de otras entidades públicas y no gubernamentales. Anticipar las necesidades y demandas de toda la población y sus problemáticas ayuda el mediador comunitario a detectar y prever los conflictos que puedan surgir y a conocer las oportunidades que pueden apoyar su resolución.

Respecto al programa de gestión de conflictos, el propósito básico de la acción gestora es el de actuar en una controversia entre personas o grupos de la comunidad. Se pretende, de esta forma, que ciertos tipos de controversias se resuelvan sin tener que llegar a un tribunal, así como que se fomente la participación de las personas en la solución de sus problemas. En el proceso de gestión de conflictos comunitarios pueden estar involucrados dos (bilateral) o mas partes (multiparte), de modo que una de las acciones iniciales del proceso de mediación comunitaria consiste en el reconocimiento y legitimación de las partes que van a negociar, así como su competencia.

Los servicios de mediación pueden apoyar la (re)construcción social potenciando el empoderamiento de los grupos, la discusión constructiva entre ellos y la superación de sus diferencias para la consecución de una mejor convivencia $y$, en definitiva, de una mayor bienestar social, finalidad última del trabajo social.

\section{Conclusión}

El proceso metodológico descrito se vincula con un trabajo social con comunidades, centrado en el modelo de desarrollo local estratégico, integral y sostenible mediante la integración de diferentes enfoques teóricos. Un trabajo social con comunidades centrado en los procesos y en las tareas de los grupos y las organizaciones comunitarias se senta en una doble dirección: está orientado a la construcción de contextos competentes (procesos), a recuperar confianzas, a desarrollar la innovación, a crear solidaridades, a generar capacidades y liderazgos endógenos, por tanto, una red de desarrollo local, y a la consecución de objetivos (tareas).

Con ello se tiene un enfoque holístico, integrador, multidisciplinar, que entrelaza estrategias y enfatiza la participación ciudadana. Por medio de la investigación-acción participativa 
de los actores implicados, en particular, y de la ciudadanía, en general, la metodología que apuesta por el cambio social sostenible parte de las necesidades sentidas. Como señala Vicente de Paula

El desafío epistemológico crítico del trabajo social en esa estructura de poder y concentración de riquezas, es el de descifrar esas condiciones generales de producción de la relación entre acumulación y política para mirar su institucionalidad concreta bajo la forma de políticas sociales, considerando las fuerzas en presencia en las arenas de poder y de la distribución (FALEIROS, 2011, p.123).

El/la trabajador/a social en la intervención colectiva adopta múltiples roles para adaptarse a los ritmos y rostros: defensor, mediador, coordinador, planificador, motivador etc. Todo ello representa la versatilidad necesaria para el profesional social interactuar con una comunidad compleja, donde el trabajo social debe "equiparse" de un discurso integrado e integrador de las características y dinámicas de la población, grupos y momentos. A la vez, con técnicas y habilidades innovadoras, y con con la lógica participativa, este profesional puede contribuir a la construcción de caminos que se encuentran sin prescribir retos infalibles, pero requieren de sus actores ser competentes en el caos del complejo universo relacional.

En resumen, el enfoque holístico, integrador, multidisciplinar, entrelazando estrategias y enfatizando la participación ciudadana, "encierra" los mejores rasgos del desarrollo de la comunidad, de la acción y de la planificación social. De los dos primeros se destaca la utilización de la organización de los ciudadanos locales para que se impliquen en la resolución de las necesidades que les afecta desde sus oportunidades y centros de interés. Y del último se sobresale el uso de los expertos y de las tareas técnicas que favorecen la investigación y coordinación de servicios/ actuaciones profesionales.

Ciertamente no podemos obviar el marco institucional del que solemos depender los trabajadores sociales, así como la percepción y expectativas que sobre nuestro trabajo tienen tanto las instituciones, los grupos y los ciudadanos, individualmente considerados. En este sentido, por una parte, nos encontramos con instituciones que enfatizan el trabajo asistencial, centrado en las personas y en las familias, en el mejor de los supuestos. Los Servicios Sociales, especialmente los de Atención Primaria, han sido, tradicionalmente, el Sistema de Protección Social desde donde se han propuesto líneas de intervención comunitaria, aunque no debemos "caer" en la tentación de considerar este ámbito ni el único ni tampoco como el mejor o el principal impulsor. Constatamos experiencias en el ámbito de la salud, de la educación, de la vivienda, del empleo-formación etc., o bien, de la integración de ellas, de enorme validez.

\section{Referencias}

ANECA. Libro Blanco para el Título de Grado en Trabajo Social. Madrid: Ministerio de Educación y Ciencia, 2005.

J. M.; CORTES, F. Trabajo comunitario, organización y desarrollo social. Madrid: Alianza Editorial, 2005.

BATTEN, T. R. El enfoque no directivo en el trabajo social de grupo y comunidad. Madrid: Euramerica, 1969.

BURGOS, M.; PÉREZ, I.; SÁNCHEZ, R. Trabajo Social en el Sistema Público de salud: Atención Primaria, Hospitalaria y salud mental. Revista de Trabajo Social y Acción Social. Málaga, edición especial, Tomo II, I Congreso de Trabajo Social, p. 7-28, abr. 2009.

CLARK, C. L. The Political Ethics of Social Work: Towards Welfare Citizenship. Basingtoke: Macmillan, 2009.

CITOLIN, A; LASKOSKI, G. A. Desenvolvimento humano: una aproximação entre os direitos humanos e a noção de desenvolvimento. Revista Emancipação. Ponta Grossa: v. 12, n. 2, p. 165-179, 2012.

CONSEJO GENERAL DE TRABAJO SOCIAL. Código Deontológico del Trabajo Social. Madrid: Consejo General de Trabajo Social, 2012.

COLOM, D. El trabajo Social sanitario en el marco de la optimización y sostenibilidad del sistema educativo. Revista de Servicios Sociales Zerbitzuan. Bilbao: v.47, n. 1, p. 109-119, 2010.

FALEIROS, V. de P. Estrategias de empowerment en trabajo social. Buenos Aires: Lumen, 2003. 
. Desafíos del trabajo social frente a las desigualdades. Revista Emancipação. Ponta Grossa: v. 11, n. 1, p. 117-128, 2011.

FERNÁNDEZ, T.; LÓPEZ, A. Trabajo social comunitario: afrontando juntos los desafíos del siglo XXI. Madrid: Alianza Editorial, 2008.

FREIRE, P. Pedagogía del oprimido. Madrid: Siglo XXI, 1972.

Pedagogía de la autonomía. Madrid: Siglo

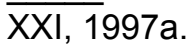

A la sombra de este árbol. Barcelona: El Roure Editorial, 1997b.

GALTUNG, J. Investigaciones teóricas. Sociedad y cultura contemporáneas. Madrid: Tecnos, 1995.

, J.; JACOBSEN, C. G. Searchin for Peace; the Road to TRANSCEND. London: Pluto. 2000.

HELLER, A. Una revisión de la Teoría de las Necesidades. Barcelona: Paidós. 1996.

HERNÁNDEZ, M.; GONZALÉZ, A.; CIVICOS A.; PÉREZ B. Análisis de las funciones del trabajo social en el campo educativo. Revista Acciones e Investigaciones sociales. Zaragoza: vol. 22, n. extra 1, p. 453, 2006.

HOLGADO, D.; MAYA-JARIEGO, I.; PALACIO, J. Diferencias en el clima para la innovación y demandas laborales en profesionales sanitarios y trabajadores sociales y práctica profesional en centros de atención primaria de salud en Andalucía. Revista Salud Uninorte. Barranquilla: vol. 28, n.1, p. 16-26, 2012.

HOLGADO, D.; MAYA, I. Preparación comunitaria y contextos de intervención: el caso de los trabajadores sociales de atención primaria en Andalucía (España). Revista Anales de Psicología. Murcia: vol. 28, n. 1. p. 150-160, 2012.

LÓPEZ, A. Profesión, ciencia y ciudadanía. Retos para el Trabajo Social y los Servicios Sociales en el siglo XXI. Azarbe. Revista Internacional de Trabajo Social y Bienestar. Murcia: vol.1, n. 1, p. 63-73, 2012.

MALAGÓN, S. La Vivienda y los Procesos de Exclusión Social. Revista Trabajo Social Hoy. Madrid: vol. extra 2, p. 101-114, 2008.

MAX-NEEF, M. Desarrollo a escala humana. Concepto, aplicaciones y algunas reflexiones. Barcelona: Icaria. 1994.

. La dimensión perdida. La deshumanización del gigantismo. Barcelona: Icaria. 2007.
NATÓ, A.; RODRÍGUEZ, M. G.; CARVAJAL, L. Mediación Comunitaria. Conflictos en el escenario social urbano. México: Universidad de Sonora-Centro Internacional de Estudios Sobre Democracia y Paz Social, 2005.

NUSSBAUM, M. Las mujeres y el desarrollo humano, Norma, Buenos Aires. 2002.

Crear capacidades: propuesta para el desarrollo humano. Barcelona: Paidós. 2012.

PASTOR, E. Participación ciudadana y gestión de las Políticas Sociales Municipales. Murcia: Editum, 2009.

Trabajo Social Comunitario. Murcia: Diego Marín. 2010 a.

Trabajo Social comunitario, participación y calidad democrática local. Dimensiones de análisis e intervención para intensificar la participación. Revista de Servicios Sociales y Política Social. Madrid: vol. 91, n. 3, p. 9-26, 2010 b.

Impact, effectiveness and sustainability of social policies and local democracy through the social involvement". Revista de Cercetare si Interventie Sociala. lasi (Romania): vol. 35, n.4, p.7- 27, 2011a.

Proximity social policies government: quality and democratic improvement analysis of local council of well being. (Murcia, Spanin). Revista de Administração Pública. Rio de Janeiro: vol. 45, n. 2, p. 377-399, 2011b.

Gobernanza de los Servicios Sociales municipales: dar voz y salida a las aspiraciones ciudadanas. Revista Cuadernos de Trabajo Social. Madrid: vol. 25, n. 1, p. 143-158, 2012.

PASTOR, E.; HUERTAS, E. La mediación penitenciaria como método alternativo de resolución de conflictos entre internos en el ámbito penitenciario. Revista Entramado. Cali: vol. 8, n. 2 p. 138-153, 2012.

PUYOL, B; HERNÁNDEZ, M. Trabajo Social en Educación. Revista Qurriculum. La Laguna: vol. 22. p. 97-117, 2009.

REID, W.J. El conocimiento aplicado a la práctica directa del trabajo social: un análisis de tendencias. Revista de Treball Social. Barcelona: vol. 169, p. 6-51, 2003.

ROBERTIS, C. D. E.; PASCAL, H. La intervención colectiva en trabajo social. La acción con grupos y comunidades. Buenos Aires: Ateneo, 1994. 
ROGERS, C. R. El proceso de convertirse en persona. Buenos Aires: Paidós, 1989.

RUIDÍAZ, C. Los Servicios Sociales Penitenciarios en la España Democrática. Revista Portularia. Huelva: vol. 4, n.1 p. 109-114, 2004.

SAAVEDRA, Ma L. (COORD.) Rol Profesional del Trabajado Social en Atención Primaria de Salud. Servicio de Atención Primaria, Planificación y Evaluación. Servicio Canario de Salud, 2004.

SÁNCHEZ, V. Programas de Prevención e Intervención Comunitaria. Barcelona: PPU, 1993.

SEN, A. Commodities and capabilities. Oxford: Oxford UP, 1987. 2000.

Desarrollo como Libertad. Madrid: Planeta,

TWELVETREES, A. Community Associations and Centres. Oxford: Pergamon Press, 1976.

Treball de comunitat. Pórtic. Institut de Treball Social i Serveis Socials. Barcelona: 1998.

Organizing for neigbourhood development: a comparative study of community bassed development organisations. Hardcover: 1996.

SUAREZ, Ma . C.; DIEGUEZ, A. J. Gestión Social en la Comunidad. Buenos Aires: Espacio, 2002. 